\title{
Unravelling the world of consciousness and opening a possible gateway for protection against radiation induced damage
}

\author{
Indraganti PK*, Namita I and Khanna A \\ Drug Repurposing and Translational Research Lab, Institute of Nuclear Medicine and Allied Sciences, Brig SK Majumdar Road, Timarpur, Delhi, India
}

\begin{abstract}
Whole body exposure to acute doses of ionizing radiation may result in either hematopoietic or gastrointestinal form of acute radiation syndrome ( $b s-A R S$ or $G I-$ $A R S)$. ARS is multifactorial in nature and is the result of complex interactions which are dynamic in a spatial-temporal fashion. This complexity poses a real challenge to radioprotective drug development endeavours (especially post irradiation protection). Understanding of the spatial-temporal complexity at cellular, tissue, organ, system and organism level, seems to be a critical prerequisite for the development of ideal medical radiation countermeasures but as of now it is not implicitly deciphered. Here, we are proposing, based on the idea of panpsychism, hierarchical consciousness (atomic, molecular, supramolecular, cellular, tissue, organ, system, systemic and so on) as a probable strategy for deciphering the complexity that evolve during and after exposure to ionizing radiation. The hierarchical consciousness is also proposed as a framework or model for artificial intelligence (AI) based strategies to understand the pathophysiology of ARS.
\end{abstract}

\section{Introduction}

For centuries the human consciousness, a state of being able to subjectively experience something, has intrigued philosophers and researchers in the deepest possible fashion [1]. The word 'consciousness' in search engines like PubMed gives around 44000 results dealing with consciousness in some or the other fashion, clearly indicating that it's an area of intense research. However, it is interesting to note that in spite of all the efforts, a consensus on consciousness is still eluding [2]. Over the past several centuries consciousness has been defined and described in a number of ways based on diverse factors including its purpose. Though the dominant theories of psychology and behaviour questions its utility, for present discussion consciousness is defined essentially assuming that it offers survival advantages to the species [3]. Consciousness can be defined as a set of abilities (by virtue of feedback loops it can generate) of an entity or a system to contemplate the future course of action using prior knowledge or framework for survival advantages or for achieving the purpose of existence. In the present discussion, consciousness will be dealt first followed by its possible role in complex manifestations like ARS. This discussion is essentially based on the following assumptions.

1. Prior knowledge (framework or memory) is essential for collapsing the wave nature and attaining the particle nature.

2. A conscious entity only can form the basis for the next higher level of consciousness.

3. The conscious entity depending upon the degrees of freedom (the different ways in which it can interact with other entities) can take up diverse forms and realization of any of the possible forms depending upon the environment that it is exposed to.

4. A certain level of consciousness would have control over its immediate lower level.

\section{The origin and evolution of consciousness}

Prior knowledge, framework or memory is essential for collapsing the wave nature and attaining the particle nature.

Quantum mechanics, the most successful theory, has undoubtedly opened up altogether a new way of looking at the universe, the quantum way. The double slit experiment, the corner stone of the quantum mechanics, is arguably one of the most beautiful experiments that were ever done [4-7]. The double slit experiment, in particular Richard Feynman's double slit experiment, demonstrated the particlewave duality of the electrons [8]. The very act of observation has been repeatedly shown to collapse the wave nature and realise the particle nature of the electron $[9,10]$. In fact, more recently studies carried out on human subjects with varied levels of concentration (concentrated attention and relaxed attention groups) both physically close as well as distant from the experimental set up have demonstrated the quantum nature of consciousness as well as the ability of consciousness (prior knowledge) to collapse the wave nature of the electrons [11-14]. The ability of the observer (whether a camera or a conscious observer like a meditating human volunteer) to collapse the wave form and realise the particle nature of an electron probably suggests that anything which has a frame work (in case of camera which is basically a collection of

${ }^{\star}$ Correspondence to: Prem Kumar Indraganti, Drug Repurposing and Translational Research Lab, Institute of Nuclear Medicine and Allied Sciences, Brig SK Majumdar Road, Timarpur, Delhi, India, Tel: 9958456999; E-mail: prem@inmas.drdo.in

Key words: consciousness, panpsychism, dark matter, big bang, apoptosis, acute radiation syndrome, radioresistance, radioprotection, artificial intelligence, quantum consciousness

Received: November 08, 2019; Accepted: November 25, 2019; Published: November 28, 2019 
atoms yet obeying the same fundamental interactions) or which can be comprehended (frame work as well as knowledge like in the case of a human observer who is aware of the experimental set up) should be able to collapse the wave nature, thus making it a reality (particle nature). In fact, Paul Davies in his book 'The Ghost in the Atom' suggested that prior knowledge is critical for collapsing the wave nature of the electrons [15].

Panpsychism, one of the interesting theories in the field of consciousness, considers that everything in this world harbours some level of consciousness and that matter has experience since $a b$ initio before it manifested as brain [16]. Atoms, molecules, supra-molecular complexes and so on, all possess a definite consciousness [17] (set of feedback loops, ability to interact with other entities). It can be proposed that fundamental particles (the quarks or even smaller entities yet to be discovered) essentially possess some consciousness which enables the realization of particle nature. It means that, for anything to be real to our minds we should have prior knowledge or necessary framework. Here framework may be defined as the necessary features which enable us to assess a space-time event or a situation. It is known that babies younger than a few months (4-7 months) do not possess the object permanence and when you hide your face, they think you cease to exist (game of peekaboo). However, babies quickly develop object permanence because they have the necessary framework to quickly realise that the object still exists even when it's hidden. Similarly, a human baby seeing a dog for the first time definitely would not be able to make out that it is a dog but nevertheless would still be able to ascertain some information about it because the baby has feedback loops to assess the dog and comprehend it. It also means, anything that is made without the requirement of the fundamental particles and their interactions or four forces (gravity, strong nuclear force, weak nuclear forces and electromagnetism) would be beyond the scope of consciousness of the entities that are made up with these fundamental particles and forces. In fact, dark matter, which forms $27 \%$ of everything and discerned indirectly from gravitational lensing, is non-luminous and probably non-baryonic in nature and was proposed to be composed of as yet undiscovered sub-atomic particles [18]. Similarly, dark energy which is composed of $68 \%$ of everything is also non-comprehensible probably because we do not have the necessary framework to collapse the wave nature and observe it as a reality. Recently, Tommy Tenkanen proposed that the dark matter may have originated before the big bang [19]. It is interesting to note that we can imagine, based on our own experiences like how explosion looks like, a stellar explosion (something made up of the same fundamental particles and the forces) somewhere at the edge of an ever expanding universe but we cannot imagine anything about the other dimensions (for example $7^{\text {th }}, 10^{\text {th }}$ ) (probably made up with a different set of fundamental particles and forces).

Test: At least there may be a few ways through which this assumption can be put to test. One way is to create a double slit experiment setup which is not comprehensible to the human volunteer (if he is not informed about the experiment) then his observation should not be collapsing the wave nature of the electron.

Test 2: Anything where a different set of fundamental forces are operating or at least where the four known forces (as of now) are not operating then it would not be possible for a camera or a meditating human volunteer (no matter how deep he may concentrate) to collapse the wave nature (of energy) and make it a reality.

\section{A conscious entity only can form the basis for the next higher level of consciousness}

With the idea of panpsychism it may be considered that the quarks are the smallest entity (as of now) with a certain level of consciousness and possess definite ways to interact with other entities leading to the formation of a next superior conscious entity a hadron (proton which is made up of 2 up quarks and 1 down quark and referred as uud or a neutron made up of 2 down quarks and 1 up quark and referred as ddu). It may be that consciousness grows progressively and an entity with a certain level of consciousness interacts with others and forms next higher level of consciousness which is not just an addition of two interacting entities but would be more than the addition of both of its constituents (at given environment or conditions). Considering that, a conscious entity can form the basis for the next higher level of consciousness and that any level of conscious entity can take up diverse forms depending upon the degrees of freedom (the number of ways it can interact with others), the realization of any of the possible forms depend upon the environment that it is exposed to. For example, hydrogen atom can interact with similar and different atoms in a variety of ways and similarly oxygen also has large but definite ways of interacting with others. However, a combination of hydrogen with oxygen (in certain stoichiometry) can lead to formation of water which has properties that are different from its individual constituents and unique to water molecules. However, it is important to note that all the different possible forms are expected to be made up of the same fundamental particles (and the basic fundamental forces) of the conscious entity from which it is formed.

An obvious question that needs to be addressed here is if nothingness cannot give rise to a conscious entity then how the most fundamental conscious unit has come into existence It may be possible to partially address this conundrum by considering the logic, discussed above, that anything where the fundamental particles and forces are of a different nature (from those we know operate in this universe) then it cannot be a reality for any conscious entity which is made up with these particles and forces. It may be possible or at least interesting to propose that a different dimension (where a different set of fundamental forces and laws operate) must have been responsible for the origin of the most fundamental conscious unit, the singularity and the big bang. In fact, some superstring theories propose the space-time during the initial phase of big bang to have 11 dimensions [20]. However, a disconnect is clearly evident here, that the idea of panpsychism does not provide a clue to how a conscious entity, obeying the standard model of particle physics, can originate from an entity which seems to be operating with a different set of physical forces. Although it is interesting to note that the ability of the dark matter to influence the matter resulted from the big bang, it indicates that dark matter might still be comprehended, or it may be a possible gateway to the other dimensions. It is interesting to note that, the researchers of Hungarian Academy of Sciences have recently reported to have found a $5^{\text {th }}$ force of nature and claims to have observed energy that connects the visible world to dark matter [21,22]. Nevertheless, with the set of fundamental forces which forms the basis of this universe, it would be difficult to comprehend information like how the big bang has started especially the events prior to the Planck's time $\left(<10^{-43} \mathrm{Sec}\right)$. However, it may be hypothesised that to understand the origin of the big bang one needs to come out with an entirely different set of fundamental forces. But the caveat here is that anything proposed on the basis of the currently known fundamental forces will be inefficient or may not suffice. It is interesting to know that various attempts to combine our two best models of physics of the universe, quantum mechanics and general relativity, into a workable whole, 
representing an essential step towards the Holy Grail of physics, "theory of everything" (TOE) remained unsuccessful [23].

The conscious entity depending upon the degrees of freedom (the different ways it can interact with similar and different entities) can take up diverse forms and realization of any of the possible forms depend upon the environment that it is exposed to.

With the assumption that consciousness progressively increases, it can be considered that a quark would have much lesser degrees of freedom (a certain number of possible interactions) when compared to a proton (uud) which may possess the next higher level of consciousness. It is well accepted that the events which happened during the first second after big bang are in a definite order (a conscious unit has given rise to the next higher level of conscious entity) and are dependent upon the conditions that prevailed (like temperature). It means, the conditions which existed within the first seconds (like the temperature etc.) in fact allowed only certain possible manifestations, resulted in quarks, antiquarks followed by electrons, protons, and neutrons and so on. It can also be argued that any change in these conditions would result in a different outcome (different conscious entities). It is noteworthy that the experiments done with particle accelerators in 1960s showed that protons and neutrons are just representatives of a family of particles called hadrons (100 hadrons detected thus far) [24]. In fact, recently, Yang et al have shown that a new state of matter, an intermediate bosonic metallic state, arises in thin film superconductors as they cooled down towards their superconducting temperatures $[25,26]$. The conditions that prevailed at different time points after the big bang, has led to the formation of the specified particles only (atom with respect to nucleus). The formation of an atom is one of the consequences of immediate lower level of conscious unit (nuclei with proton) but it may not be the only fate. It is possible that other probabilities which are possible at the prevailing conditions might have taken place, but the consciousness of the next higher level might have determined its progressive fate in that environment. Contrary to the general thinking that only a specific event (like proton and neutrons forming nuclei and electron orbiting the nucleus) has taken place, it is likely that a conscious unit can take up any number of different outcomes. It is known that a stable atom requires an electron circling the nucleus and if the electron shots into the nucleus then there won't be any formation of a stable atom. However, it does not mean that it is the only outcome, it may have been the only successful (in terms of stability and diverse forms it can take in the given conditions) outcome. All other possibilities must have happened, and it must have taken forms which are entirely different from an atom. It is obvious to think that any deviation in the plan of an atom should lead to no formation of a stable atom. It is interesting to note that recently Henderson James Cleaves et al have suggested that DNA, the blueprint of life, is just one of the million possible genetic molecules [27]. Similarly, among the total number of compounds that can be formed by all the elements known so far (118 elements are there in periodic table) the contribution of carbon is much more than the others. It indicates that carbon is more conscious than the rest of the elements. But it may be so that in this environment, carbon is able to achieve the diversity (due to catenation property), that may not be optimal for other elements to realize their complete potential. That means in other environmental conditions they might achieve much more diversity. It also indicates that it's not the size of the atom that determines the level of consciousness, but it is the capacity of an atom to optimally diversify in a given environment. With the idea that consciousness has the intrinsic ability to diversify depending upon the environmental conditions, it could be argued that anything which is confined to relatively homogenous milieu should exhibit lesser diversity (in terms of appearance and consciousness). Microbes like E. coli which survive in relatively smaller space-time (though they don't grow old and just divide typically every 20 minutes) don't experience any significant change in their milieu and they all look typically same. Similarly, a group of penguins (a rookery) and a number of other animals look alike probably because they experience relatively similar environments. With the same logic, anything which has a life span long enough to experience different seasonal changes and other environmental changes is likely to exhibit a greater diversity. Unlike a rookery or a shoal, we humans look different [28] with possibly different degrees of consciousness. The ability to reproduce any time of the year along with different environments we live in, may probably be a contributory factor, apart from the genetic component, in diversification of the consciousness.

Test: In different environmental conditions any of the other elements should be able to form more compounds than carbon.

\section{A certain level of consciousness would have direct control over its immediate lower levels}

The discussion presented here is different from the ambiguous word complexity from the point that it is the hierarchically increasing consciousness which is at work. It may be that a certain level of consciousness is under the control of immediately higher level of consciousness and that means if you consider conscious levels of $1,2,3$, 4 then level 2 is under the control of 3 and not 4 or 1 . In order to exert control over 2 , the level 4 needs to go through 3 . In other words, only molecules can bring out any change that is warranted at the atomic level rather than the tissue or organ. Similarly, system controls the organs, organ control tissues, tissues control cells, cells control sub-cellular entities and so on.

The idea of hierarchical consciousness may also be able to address some of the most intriguing aspects of human endeavours. Following are some of them which have been discussed based on the idea of hierarchical consciousness.

\section{Origin of universe and consciousness-The panpsychism}

All the models that have been proposed regarding the origin of universe fall broadly into two categories. Universe has generated from nothing and the other one that there is no beginning. Currently the big bang theory is the most accepted theory and it is possible to trace it up to a scale of a millisecond after the big bang and possibly up to the Planck's time $\left(10^{-43} \mathrm{sec}\right)$. It seems how small one can go back in time depends upon the time scale at which the most fundamental consciousness operates. In other words, sub cellular entities like enzymes, cells, tissues, organs, systems, organism work in a time scale of increasing order. Similarly, atoms function at a time scale of $10^{-12} \mathrm{sec}$ while sub-atomic particle like proton at $<10^{-14} \mathrm{sec}$, and other fundamental particles at even lower time scales (quarks etc.). Anything that operates in time scales smaller than the time scale of most fundamental conscious unit would be incomprehensible and cannot be a reality. With instrumentation and mathematical formalism, based on the fundamental forces, we think the big bang is the starting point. But it might be possible that at even smaller time scales the origin can be traced back. So, it seems the beginning beyond the Planks era may be a possibility (the tunnel between the planks time and 0 may be longer than one can imagine). Although, it is our limitation posed by the fundamental consciousness (the most fundamental entity working at tiny time scales). So, some entity which works at much smaller time scales (< our lower limit) is the one which probably has given rise to the big bang. The "problem of 
time" - is that time may have a different meaning in quantum mechanics and general relativity, and this is best exemplified by the WheelerDeWitt equation [29]. Their attempt to unify relativity and quantum mechanics resulted in time essentially disappearing completely from their equations, suggesting that time does not exist at all and that, at its most fundamental level, the universe is timeless.

\section{Panpsychism- The problems and hierarchical consciousness- The possible solution}

Until the middle of $20^{\text {th }}$ century, the idea of panpsychism was a default theory in the philosophy of mind. However, the panpsychism suffers from a major problem that any attempt to introduce the consciousness to originate from an inert or non-conscious matter would mean going against the fundamental construct of science. David Chalmers said it is beyond possible to visualize the existence of consciousness from nonconscious matter [30]. As a theory 'consciousness gave birth to matter' assumes that universe is alive. However, it can be argued that being alive and being conscious are not the same. Consciousness is required and forms the basis for conscious life to emerge. It can be said that life is an advanced state that consciousness can take up or shape up to (it is just one of the different ways a conscious entity could evolve into-discussed below).

\section{The hard problem of consciousness}

The problem of explaining the relationship between the abstract relational properties of fundamental particles like spin, mass, momentum and charge and the feeling of experiences (like seeing a bunch of Fosteriana tulips) is one of the major difficulties for the idea of panpsychism. This is known as the hard problem of consciousness [3133]. If one tries to deduce a relationship between the arrangement of atoms and the warmth of fire during a cold night, then there is a discord as arrangement of atom is insufficient for higher level of consciousness (the feeling of warmth). The arrangement of sub-atomic particles definitely forms the basis for the next higher level of consciousness, and it elevates in a progressive fashion (atomic, molecular, macromolecular, organelle, cellular, tissue, etc.). However, the point that is being tried to put forward in this discussion is that the human consciousness is progressive in nature rather a combination of subjective inner lines. That is the human consciousness, which is generally defined as the feeling of what it is like, to subjectively experience something, may not be the result of countless physical particles that make up the nervous system but a hierarchical increase in the consciousness that ultimately gives rise to human consciousness.

\section{The combination problem}

Another problem with panpsychism is that there is no-coherent non-magical way in which lower level (of subatomic particles) subjective point of view could combine to form a higher level of subjective point of view as we all experience. This is called the combination problem [34] and it may be addressed by considering that it is the hierarchical increase in the consciousness that is responsible for the subjective experiences rather than the combination of subjective point of views, leading to human consciousness. In other words, a level immediately lower to human consciousness is responsible for the evolution of human level consciousness. It is noteworthy that unlike lower levels of consciousness (like atomic, molecular, cellular, system and systemic) the human consciousness is amenable to anaesthetics. The space-time has a consciousness but at least it is different from the human consciousness in terms of degree. A rock with a collection of atoms is more conscious in comparison to its constituents but it does not even come close to match the consciousness of a macromolecule like protein (which is also non-living). A rock can never transform into a living thing even though it is made up of the same fundamental atoms and forces as we do, because atoms with certain level of consciousness and with certain degrees of freedom evolved in a certain way (a rock way). Probably for a rock to evolve into higher consciousness, like that of a protein, it needs to reverse to the level of atoms and then take the course that results in the formation of a protein. It can be anticipated that it is much more difficult than the origin of life as it needs to traverse a longer path.

\section{Consciousness as a physical entity}

A number of researchers have been trying to identify and quantify consciousness [35-37]. Gottfried Leibniz, a seventeenth century thinker, famously said that if we could blow the brain up to the size of a mill and walk about inside, you would not find consciousness. In fact, other philosophers argued that we do not have enough mental fire power to solve this as a system can never understand about itself. In fact, it can be argued here that a system would definitely fail to understand itself if we consider that human consciousness, the subjective experience or feeling unique to all of us, is ultimate and the only consciousness. However, as discussed throughout this article, the human consciousness is the resultant of a hierarchical and progressively complex consciousness and we may have enough mental fire power to comprehend all the levels of consciousness barring the ultimate consciousness. An interesting question that pops up here is about the nature of ultimate consciousness. Probably, a group of people (like family consciousness) is higher to an individual human consciousness and community consciousness should be possessing enough mental fire power to understand the human consciousness and so on. Ultimately, it seems that the consciousness of all humans of the planet earth may be the highest consciousness which should be having the power to understand all the levels that are preceding. It seems consciousness is very unlikely to be identified as a physical entity and probably the simplest way to realize consciousness is to consider it as the wave front that one can see during the domino effect which is not physically linked to any of the falling dominos but a cumulative effect which manifests with the falling dominos.

\section{Life is one of the many forms consciousness can shape to}

Based on the discussion presented above, it may be argued that consciousness can take up variety of forms and in fact, all the diversity and the complexity that is evident in the microbial world, in flora and fauna may be explained by considering that a conscious entity can take up diverse forms depending upon the environmental conditions. The life which was defined in a number of ways but is essentially described through a set of features like ability to maintain homeostasis, reproduction, metabolism etc. [38-40]. Atoms with a set of feedback loops can interact with other or similar types to form molecules which in turn can take up different forms depending upon molecule's level of consciousness (feedback loops). It may be that the conditions which evolved several billions of years after the big bang (comparatively low temperatures, lightening, hydrothermal vents etc.) were conducive for a conscious entity (molecules, supramolecular complexes etc.) to evolve into an entity which gained the self-replicating ability. In fact, recently, in laboratory conditions, a self-assembling peptide has been synthesised $[41,42]$. However, the point that is being tried to put forward here is that, provided the conditions are conducive, a variety of conscious forms should have originated depending upon the environmental conditions. Infact there are evidences in this direction [43-45]. It is important to note that in spite of different theories like the primordial soup, chemical evolution, metabolism first, and the RNA world, the DNA world is the 
most successful. It can be argued that the DNA based consciousness probably offers the most optimal way to evolve and diversify.

One of the intriguing questions about the origin of life is, did it originate just once? $[46,47]$. With the idea of hierarchical consciousness, it is possible that newer life forms may be originating and in fact evidences are accumulating in support of it $[48,49]$. In fact, it seems easier to explain the perceivable universe and its functioning considering the hierarchical consciousness and its evolution by chance rather than considering involvement of an intelligent designer. Albert Einstein in one of the letters to Max Born in 1926, while referring to quantum mechanics wrote 'I am convinced that $\mathrm{He}$ does not play dice'. "He" Einstein refers to is God. It's difficult to predict whether He (god) plays dice or not but definitely he has the time to, because at least the solar system and part of the cosmic web, which we can sense, can be explained by hierarchal and progressive consciousness and is selfsustaining without any steering. It's like presidential solicitation is not required in routine county affairs.

\section{Hierarchical consciousness and homeostasis}

As discussed above, at every level, the consciousness, like a flowing river, has propensity to diversify depending on the conditions. Even the inanimate entities like rocks also change some of their features with time when left in open. It is interesting to note that all the living organisms from the most primitive life that emerged in primordial soup to the most complex multicellular organisms that ever existed and existing in principle achieve internal orderliness probably at the cost of entropy of the milieu or outside world [50,51]. The most primitive life forms also exhibit orderliness or homeostasis. Through natural selection, all life forms have evolved diverse strategies with one important function, to keep the internal milieu constant [52]. The phenomenon of homeostasis seems to have a definite advantage that it puts the consciousness in control and probably curbs it from diversifying. It can be argued that unlike sedentary organism, like plants, the mobility of comparatively advanced life forms, come with both advantages and also the disadvantages (like manoeuvring from safe zones to territories infested by predators). The ability to plan for the future, in a sense, also seems to be a part of the repertoire to maintain the homeostasis. Issues like whether animals possess consciousness, or do they plan for future have been and are being intensely debated [53-55]. Keeping the paramount importance, the homeostasis holds, it is interesting to propose that all the conscious entities probably plan for future (it seems it is an intrinsic character). However, it is obvious that the nature of planning would be different, and it can range from resisting a change to advanced future planning that human practice. Plants certainly do not plan the way animals or humans do but they must be having strategies to safeguard their future. It is noticeable that many of the human diseases are the resultant of changes in micro- or macro-milieu [56,57]. It may even be argued that complex pathological manifestations like ARS, cancer and normal ageing is also because of changes in the micro- or macro milieu which result in diverse levels of consciousness to evolve differently (for example evolving to a consciousness level which is commonly referred as tumorigenic).

\section{Possible role of consciousness in radiation induced ARS}

\section{Massive apoptosis in acutely exposed organism}

A cell with certain feedback loops has been evolved to perform a set of functions in the present and probably does not plan the future (cellular consciousness: It is the sum of all the activities that a cell as an entity could do). It has not been evolved (not conscious enough) to have a set of capabilities that can foresee the future. Unlike a cell, a human being with a large number of feedback loops (of course by virtue of having the neocortex) is equipped with the ability to plan for future based on previous memories/experiences [3]. It is noteworthy that this advanced consciousness is nothing but a sum of all the levels of consciousness that work at different levels in our body (subcellular, cellular, tissue, organ, system and organism levels). Apoptosis, one of the cellular fates, has been well attributed to the manifestation of ARS in radiation over exposed victims [58,59]. Apoptosis or programmed cell death is a cellular phenomenon and every cell has the machinery to execute and in fact, a number of cell types (like rapidly proliferating cells) readily turn this programme on in response to a wide variety of stimuli including damage to the DNA and other macromolecules. From the point of evolution, apoptosis serves the purpose of weeding out the cells with irreparable DNA damage which contributes to genomic instability and increases the proclivity for induction of cancers [60,61]. As long as the apoptosis is under control, it offers an advantage to the species. However, the problem pops up in organisms exposed to acute doses of ionizing radiation, when a large number of cells turn on the apoptotic programme either in response to the irreparable DNA damage or to the changes in micro- and systemic- milieu (protracted oxidative stress and inflammation). As this apoptotic wave results in massive loss of cells of primarily radiosensitive tissues like bone marrow, spleen, thymus that leads to hematopoietic depression and directly impacts post-irradiation recovery [62]. Even though apoptosis serves to maintain long term genomic stability but the massive loss of cells without the apparent realization of its immediate impact seems to be an issue. It seems the cells, with the power to execute apoptosis, are unable to sense the overall impact of apoptosis at tissue and higher levels. Assessing the overall impact of massive apoptosis is beyond the realms of the cellular consciousness which seems to be a privilege of higher conscious entities like tissue or organ or organism. A simple real-world approach that many of us would follow may help in better understanding of the point that is being tried to project here. Often, we plan in such a way that, keeping the long-term advantages in mind either we sacrifice or ignore the immediate apparent benefits. However, in case of emergency, the concept of long-term advantage takes the backstage and appropriate response which serves the immediate requirement takes over (in a way this approach also has a long-term advantage as for seeing the future one needs to survive today). We as an organism enjoy this level of consciousness for addressing a situation which seems to be missing at cellular consciousness level that is meant for catering the immediate needs.

Unlike multicellular organisms, single cell organism like bacteria (especially the solitary species) does not readily undergo apoptosis $[63,64]$. In fact, it is counterintuitive from our understanding of evolution through natural selection that a single cell organism like bacteria should undergo apoptosis as it serves no long-term purpose from the point of bacterium [65]. It is interesting to note that certain bacteria operate the SOS repair system which is highly error prone, and it operates with the sole intention of immediate survival over the possible long-term genomic instability $[66,67]$. However, as mentioned earlier a given level of consciousness would be under the control of immediately higher level of consciousness. This hierarchical relationship should be evident in living organisms. In case of colonizing bacteria, which is made up of individual bacteria and acts as one conscious unit (community conscious), and the fate of individual bacteria should be under the control of the next higher conscious entity (community consciousness). In fact, the bacterium should be undergoing apoptosis or similar mechanisms leading to self- killing, provided its elimination 
offers advantage to the overall survival of the community. In fact, $E$. coli which is a colonizing bacterium has requisite cellular machinery to execute apoptosis. It has been reported that $E$. coli has maz E (which encodes for an antitoxin) and maz F (which codes for a toxin) system along with Rec A system which can initiate apoptosis in response to heavy DNA damage $[68,69]$. In fact, maz E/F system inhibits Rec A mediated apoptosis through EDF. It is interesting to note that EDF is an external factor which is released by other bacteria in the community through quorum sensing. Basically, colony consciousness is controlling the cellular activities and fate. In this case both Rec A and maz E/F are cell death inducers. maz E/F in presence of EDF induce apoptosis and also inhibit RecA pathways [68]. It could be suggested that in $E$ coli, $\operatorname{Rec} A$ is its intrinsic pathway which is a part of cellular consciousness while maz $\mathrm{E} / \mathrm{F}$ is community consciousness pathway controlling the individual's fate (in this case induction or inhibition of apoptosis) [70]. This Rec A and maz E/F also gives an idea how a higher consciousness regulates the immediate lower entity. In fact, similar hierarchical regulation between different levels of consciousness should also be working in more evolved biological systems like mammals and humans. It can be assumed that in order to execute the control over its immediate lower conscious entity it must have the machinery like receptors for sensing the signals. In fact, human cells also exhibit multiple pathways controlling the regulated cell death (apoptosis-extrinsic and intrinsic, pyroptosis ferroptosis, parthanotos, entotic cell death, NETtotic cell death, autophagy-dependent cell death (ADCD), immunogenic cell death (ICD), lysosome-dependent cell death (LDCD) and other forms of cell death) [71]. It may be possible, like in bacteria, these different forms of cell death are actually the mechanism through which higher level of consciousness exert their control over the fate of lower conscious units (Intrinsic pathway are a part of the cellular consciousness while other forms of cell death are a part of the higher levels of consciousness). It is interesting to test whether like maz E/F system in bacteria how different forms of apoptosis influence each other. It is noteworthy that evidences are accumulating that similar cross talk between different forms of cell death does occur in advanced multicellular systems [72].

Based on the hierarchical regulation that operates in multicellular organism, it can be anticipated that some level of consciousness should be able to assess the impact of massive apoptosis in hematopoietic organs like bone marrow, spleen and thymus and probably should prevent apoptosis keeping the immediate survival in mind. However, unlike human pathologies like diabetes, hypertension, bacterial or viral infections, whole body acute radiation exposure is different from the point that it inflicts damage to a variety of systems in parallel [73]. Irrespective of the part of the body, ionizing radiation induced damage results in a response at organism, systemic, systems, organ, tissue, cellular, sub cellular macromolecule and molecular, atomic level aimed at restoring the homeostasis. It seems it is defying involvement of any higher level of consciousness putting survival of the organism ahead of genomic stability. It may be possible that, like in bacteria, organism exposed to acute dose of radiation may be a disadvantage to the probable long-term survival of the immediate family or community. In view of this, it is interesting to explore the possible influence of consciousness, superior to individual organism's consciousness like immediate cage mates (in experimental animals) or, the immediate family and community, in case of humans, which forms the superior conscious entity on recovery of exposed victims. In fact, empathy which works at individual level is a well-established phenomenon [74-76].

\section{Consciousness and radiosensitivity}

Different organism's exhibit varied sensitivity or tolerance to radiation over exposures and it ranges from extreme form of radio tolerance to extremes of being sensitive to it. One of the well-established laws of classical radiobiology, 'law of Bergonié and Tribondeau, says that rapidly dividing cells are radiosensitive and terminally differentiated cells exhibit radio resistance. E. coli which divides approximately every 20 minutes is way more proliferative than a typical mammalian cells with a division time approximately $24 \mathrm{~h}$. However, E. coli exhibits a greater tolerance to radiation induced lethality than a mammalian cell. This anomaly has been attributed to the differences in the genome size [77]. Although, it is interesting to delve in the role of consciousness of the organism in its radio-tolerance. It may be that increase in the number of hierarchical consciousness is inversely related to the radiosensitivity of the organisms. A typical colonizing bacteria, like E.coli, which is connected to the other bacteria of the colony through quorum sensing, seems to be under the regulation of lesser number of conscious levels (atomic, molecular, supra molecular, organelle, cellular, community) when compared to a cell (like a lymphocyte) in a mammalian system. However, this raises the question that probably the comparison between a bacterium and a mammalian cell which are clearly different in their consciousness may not be appropriate. It is interesting to note that a protein from a bacterium and a mammalian cell with similar consciousness, should not be drastically different from radiosensitivity point of view (with an end point like protein dysfunction). Based on this idea it can be suggested that a lower conscious entity cannot mimic the functioning of a higher conscious entity because it is beyond its realm to predict the functioning of higher consciousness. It may that the radioprotective strategies employed by lower conscious entities (like tardigrades, archaebacterial, insects, nematodes, arthropods, lower mammals etc.) may not be translatable or useful to higher conscious entities (like humans). In fact, the translatability of the data generated based on the experimental animals like rodents, non-human primates are an area of intense debate [78]. It is also well recognised that irrespective of the data generated with experimental animals, the clinical trials data is mandatory and forms the basis for drug approvals. Even the variation that is being observed with any drug in terms of its efficacy and at times safety in different ethnic groups and also the need for personalized medicine may be explained by considering the intrinsic diversifying potential of consciousness (all human are different by the virtue of having different levels of consciousness).

Another level of consciousness which may have a relationship with radio sensitivity of an organism is the feature of motility. Based on the kinesis, cells of a multicellular organism can be broadly divided in to two main types, cells which are sedentary and cells which can move. From radio sensitivity point of view cells with ability to move (higher level of consciousness when compared to cells which are static) seems to be more sensitive. Cells of the connective tissue (blood) like hematopoietic stem and progenitor cells, lymphocytes, neutrophils intestinal epithelial cells are sensitive to radiation and form some of the earliest cells types to get eliminated through apoptosis after irradiation. The extreme radio sensitivity exhibited by terminally differentiated lymphocytes and their ability to readily undergo interphase cell death has intrigued researchers for a long time which still remain poorly explained [79]. It may be suggested that it could be due to their ability to move which makes them a higher conscious entity and thus radiosensitive. Similarly, cells which form the framework of the tissues like muscle, heart, kidney, liver, brain etc. are radio-tolerant probably because they belong to comparatively lower conscious level. Apparently, this division fails to address the radio-tolerance feature exhibited by other cells types (RBC, platelets) which are a part of the connective tissue and possess motility. Evolutionarily, kinesis seems to be associated with apoptosis. The need may root from the fact that consciousness has the intrinsic 
ability to diversify and as the motile cells gets exposed to different microenvironments there exist a propensity for change. Probably loss of nucleus, expulsion of organelles in case of RBCs [80] a particular life span and strong control over regulated cell death in case of lymphocytes seems to be an evolutionary adaptation for restricting the mobile cells from diversifying. It is noteworthy that epithelial-mesenchymal transition (EMT) which involved gaining of motility has shown to play a role in the tumorigenic process [81].

\section{Targeting consciousness for protection against radia- tion induced damage}

The problems associated with acute and chronic radiation exposure has long been realized [59]. A large number of chemicals of both natural and synthetic origin have been tested for radioprotection [82]. However, a safe and effective radioprotector for human application still remains an unmet need. A number of researchers are actively engaged in synthesis, screening and identifying novel molecules for prophylactic and mitigative potential $[83,84]$. These efforts are based on the hope that the screening endeavour would lead to identification of a molecule with a potential to render protection to an organism from whole body acute radiation exposure. Keeping both the spatial-temporal complexity exhibited by different levels of consciousness (cellular, tissue, organ, systemic) it seems an effective drug should possess magical properties. It might include properties like differential pharmacokinetics in a temporal fashion, with multiple properties (free radical scavenging, modulator of apoptosis and inflammation and so on) which are manifested in a spatial-temporal fashion and must be available in the system for weeks together exerting their action during the course when required and ceasing to act when not. It is obvious that such a 'wonder drug' may not be possible or at least cannot to be realized in any time soon. It may be argued that the need of the hour is probably better understanding the complexity associated with the ionizing radiation and involvement of different levels of consciousness and their flow. At present more than 10000 drugs belonging to different chemical classes are available for managing a variety of clinical indications [85]. It is noteworthy that the indications (like oxidative stress, inflammation, opportunistic infections, hematopoietic depression etc.) in isolation or in combination have shown to be responsible for a variety of pathological scenarios. In case of acutely exposed organisms, these and many more indications appear on the landscape of tissues in temporal fashion. It can be argued that we already have potent drugs and probably generating the know how about using them in combination or in a temporal fashion (right drug at the right time) is the need of the hour. Deciphering the hierarchical consciousness and its role in acute radiation syndrome may be a promising target for effectively countering ARS and realizing ideal radioprotection and mitigation.

\section{The hierarchical consciousness a model for artificial in- telligence for understanding ARS}

The extreme complexity associated with the whole-body acute radiation exposures is a well-established phenomenon [86]. Understanding of the complex interactions that comes on to the landscape of tissue, organ and system of the exposed victim is critical for devising ideal radioprotective strategies [87]. Though life evolved comparatively more recently (around 4 billion years ago), it is the result of almost 13.8 billion years of evolution without abeyance. Not surprisingly the human biology exhibits a staggering complexity and understanding to its core is a real challenge. One of the possible strategies for understanding the complexity associated radiation exposure may be to resort to the artificial intelligence [87]. Artificial intelligence has been contemplated to radically change the way in which we make predictions about a variety of phenomenon including automation, climate changes and healthcare $[88,89]$. In fact, AI has also been predicted to be useful in understanding the normal as well as the pathophysiology associated with radiation over-exposure [87]. However, a model is essentially required for developing a framework on which an AI would work with an ultimate aim to understand the human biology. One of the first step to understand the complexity associated with any system is to divide it into smaller units. As this sub-division of a system will greatly facilitate to figure out the possible interactions between different units ultimately leading to a systems response. However, the difficulty with this is to identify the most effective way in which the system can be subdivided into smaller units. As discussed in this article, the consciousness might be an effective way to build this framework. The hierarchal consciousness is proposed to be a more effective way to understand the flow of information and the manner in which different levels of consciousness seep into the cellular, tissue and system's landscape. However, from practical point, the hierarchal consciousness offers an advantage that it still helps in subdivision without seriously compromising the end result. For example, the simple division of increasing consciousness may be cellular, tissue, organ, system, systemic and organism. Of course, more effective division would be the one based on our current understanding of homeostasis [90]. In case of radiation exposure, supramolecular damage forms the level 1 consciousness and next conscious level would be all the sensors (can be local or circulatory) which are capable of sensing the damage, next would be all the mediators which bring in the response, and so on. It is clear that this division is far from ideal and with better understanding of different aspects of homeostasis it can be anticipated that this division would be more effective from the point of devising a framework for an AI to work on it and understand the pathophysiology of ARS.

\section{Conclusion}

The discussion presented in this article is essentially based on the idea of panpsychism and it proposes the origin and evolution of a progressive hierarchical consciousness. This might prompt many to think that the complexity that is evident post-acute radiation exposures has just been referred as 'consciousness. However, it is different from the all-inclusive and often ambiguous word complexity from the point that it is a hierarchically increasing consciousness which is at work and understanding of the flow or regulation of it would enable us to make predictions about macro as well as micro systems and devise countermeasures against complex pathologies like ARS. Keeping the ongoing endeavours like human brain project, brain research through advancing innovative neurotechnologies (BRAIN), human connectome project, human metabolome project, human proteome projects and quantum computing based artificial intelligence it is interesting to contemplate that understanding of hierarchical consciousness based human biology in health and disease should not be a dream but a definite reality. After all, as Joseph Conrad, a $19^{\text {th }}$ century writer, once said "The mind of a man is capable of anything... because everything is in it, all the past as well as all the future".

\section{Acknowledgement}

Authors gratefully acknowledge the support from Defence Research and Development Organization (DRDO), Delhi, India. Authors acknowledge the manuscript reading and suggestions by Prof. SP. Bedi (Ex professor, English department, PGDAV, University of Delhi), Dr. Deepak Sehgal (Deen Dayal Upadhyaya College, Delhi 
University, Delhi, India) and Dr. Archana Sehgal (Guru Gobind Singh Indraprastha University, Delhi, India).

\section{References}

1. Seth AK (2018) Consciousness: The last 50 years (and the next). Brain and Neuroscience Adv 2: 1-6.

2. Chalmers DJ (2013) Panpsychism and Panprotopsychism. Amherst Lect in Phil: 8.

3. Kagan J (2014) Review: the future of the mind: the scientific quest to understand, enhance, and empower the mind. Cerebrum 2014: 7. [Crossref]

4. Crease RP (2002) The most beautiful experiment. Phys World: 15.

5. Thomas Y (1802) The Bakerian Lecture: On the theory of light and colours. Phil Trans $R$ Soc Lond 1802: 12-48.

6. RP Feynman, R B Leighton, M Sands (1963) The Feynman Lecture on Physics (AddisonWesley) vol 3 ch 37 (Quantum behaviour),

7. Howie A, Fowcs Williams JE (2002) Interference: 200 years after Thomas Young's discoveries. Philos Trans R Soc Lond 360: 803-1069

8. Bach R, Pope D, Liou S, Batelaan H (2013) Controlled double-slit electron diffraction. New J Phy 15: 033018.

9. Aharonov Y, Cohen E, Colombo F, Landsberger T, Sabadini I, et al. (2017) Finally making sense of the double-slit experiment. Proc Natl Acad Sci U S A 114: 6480-6485.

10. Coles P, Kaniewski J, Wehner S (2014) Equivalence of wave-particle duality to entropic uncertainty. Nat Commun 5: 5814. [Crossref]

11. Radin D, Michel L, Johnston J, Delorme A (2013) Psychophysical interactions with a double-slit interference pattern. Phys Essays 26: 553-566.

12. Radin D, Michel L, Galdamez K, Wendland P, Rickenbach R (2012) Consciousness and the double-slit interference pattern: six experiments. Phys Essays 25: 157-171.

13. Radin D, Nelson R (1989) Evidence for consciousness-related anomalies in random physical systems. Found Phys 19: 1499-1514.

14. Vujanac A, Srejovic I, Zivkovic V, Jeremic N, Jeremic J, et al. (2019) Quantum nature of consciousness-Double slit diffraction experiment in medicine. Med Hypotheses 133: 109382. [Crossref]

15. Davies PCW, Brown JR (2004) The ghost in the atom: A discussion of the mysteries of quantum physics. Cambridge university press.

16. Morch HH (2017) Is matter conscious? Why the central problem in neuroscience mirrored in physics. Nautilus.

17. Bailey A (1922) The consciousness of the atom. A series of lectures delivered in New York city, winter of 1921-22.

18. Spergel DN (2015) The dark side of cosmology: dark matter and dark energy. Science 347: 1100-1102. [Crossref]

19. Tenkanen T (2019) Dark Matter from Scalar Field Fluctuations. Phys Rev Lett 123: 061302. [Crossref]

20. Petr H, Edward W (1996b) Eleven-dimensional supergravity on a manifold with boundary. Nuclear Phy: B475: 94-114.

21. Krasznahorkay AJ, Csatlós M, Csige L, Gácsi Z, Gulyás J, et al. (2016) Observation of Anomalous Internal Pair Creation in ${ }^{\wedge}\{8\}$ Be: A Possible Indication of a Light, Neutral Boson. Phys Rev Lett 116: 042501.

22. Johnson S (2019) The â $€^{\sim} X 17 a ̂ €^{\mathrm{TM}}$ particle: Scientists may have discovered the fifth force of nature. BigThink.

23. Weinberg S (2011) Dreams of a Final Theory: The Scientist's Search for the Ultimate Laws of Nature. Knopf Doubleday Publishing Group.

24. Povh B, Rith K, Scholz C, Zetsche F (2008) Particles and Nuclei: An Introduction to the Physical Concepts. Springer.

25. Yang C, Liu Y, Wang Y, Feng L, He Q, et al. (2019) Intermediate bosonic metallic state in the superconductor-insulator transition. Science 366: 1505-1509.

26. Brown University (2019) New state of matter: A cooper pair metal. ScienceDaily 14 November.

27. Cleaves HJ, Butch C, Burger PB, Goodwin J, Meringer M (2019) One Among Millions: The Chemical Space of Nucleic Acid-Like Molecules. J Chem Inf Model 59: 4266-4277. [Crossref]
28. Sheehan MJ, Nachman MW (2014) Morphological and population genomic evidence that human faces have evolved to signal individual identity. Nat Commun 5: 4800. [Crossref]

29. DeWitt BS (1967) Quantum theory of gravity. I. The canonical theory. Phys Rev 160 1113-1148.

30. Chalmers D (1995) Facing up to the hard problem of consciousness. J Conscious Stud 2: $200-219$.

31. Dennett DC (2018) Facing up to the hard question of consciousness. Philos Trans $R$ Soc Lond B Biol Sci: 373. [Crossref]

32. Nagel T (1974) What is it like to be a bat? Philos Rev 83: 435-450.

33. Chalmers DJ (2017) The combination problem in panpsychism in Panpsychism Contemporary perspectives. Oxford University Press, New York, USA.

34. Crick F, Koch C (2003) A framework for consciousness. Nat Neurosci 6: 119-126. [Crossref]

35. Casali AG, Gosseries O, Rosanova M, Boly M, Sarasso S, et al. (2013) A theoretically based index of consciousness independent of sensory processing and behavior. $\mathrm{Sc}$ Transl Med 5: 198ra105.

36. Stender J, Mortensen KN, Thibaut A, Darkner S, Laureys S, et al. (2016) The Minimal Energetic Requirement of Sustained Awareness after Brain Injury. Current Biol 26 : 1494-1499.

37. Seth AK, Izhikevich E, Reeke GN, Edelman GM (2006) Theories and measures of consciousness: an extended framework. Proc Natl Acad Sci U S A 103: 10799-10804. [Crossref]

38. Gazzaniga MS (2019) Following Schrödinger's Code: A Personal Journey. J Cogn Neurosci 31: 1777-1781. [Crossref]

39. Benner SA (2010) Defining life. Astrobiology 10: 1021-1030. [Crossref]

40. Anet FA (2004) The place of metabolism in the origin of life. Curr Opin Chem Biol 8 : 654-659. [Crossref]

41. Lee DH, Granja JR, Martinez JA, Severin K, Ghadiri MR (1996) A self-replicating peptide. Nature 382: 525-528. [Crossref]

42. Morrow SM, Colomer I, Fletcher SP (2019) A chemically fuelled self-replicator. Nat Commun 10: 1011. [Crossref]

43. Raup DM, Valentine JW [Crossref] (1983) Multiple origins of life. Proc Natl Acad Sci U S A 80: 2981-2984.

44. Schulze-Makuch D, Wagner D, Kounaves SP (2018) Transitory microbial habitat in the hyperarid Atacama Desert. Proc Natl Acad Sci U S A 115: 2670-2675. [Crossref]

45. Maruyama S, Kurokawa K, Ebisuzaki T, Sawaki Y, Suda K, et al. (2019) Nine requirements for the origin of Earth's life: Not at the hydrothermal vent, but in a nuclear geyser system. Geosci Front 10: 1337-1357.

46. Theobald DL (2010) A formal test of the theory of universal common ancestry. Nature 465: 219-222. [Crossref]

47. Steel M, Penny D (2010) Origins of life: Common ancestry put to the test. Nature 465 168-169. [Crossref]

48. Life may have emerged not once, but many times on Earth. 17 August 2016. New Scientist. Avaialible from: https://www.newscientist.com/article/mg23130870-200life-evolves-so-easily-that-it-started-not-once-but-many-times/\#ixzz65bkbBiB6

49. Weiss MC, Sousa FL, Mrnjavac N, Neukirchen S, Roettger M, et al. (2016) The physiology and habitat of the last universal common ancestor. Nat Microbiol 1: 16116 [Crossref]

50. Egel R (2014) Origins and emergent evolution of life: the colloid microsphere hypothesis revisited. Orig Life Evol Biosph 44: 87-110. [Crossref]

51. Egel R (2012) Life's Order, Complexity, Organization, and Its ThermodynamicHolistic Imperatives. Life (Basel) 2: 323-363.

52. Cannon WB (1932) The wisdom of the body. WW Norton \& company, Inc. New York, USA.

53. Cambridge Declaration on Consciousness. Animal Consciousness Officially Recognized by Leading Panel of Neuroscientists. 3 September 2012. Available from: http://fcmconference.org/img/CambridgeDeclarationOnConsciousness.pdf

54. Boly M, Seth AK, Wilke M, Ingmundson P, Baars B, et al. (2013) Consciousness in humans and non-human animals: recent advances and future directions. Front Psychol 4: 625 . [Crossref] 
55. Proctor HS, Carder G, Cornish AR (2013) Searching for Animal Sentience: A Systematic Review of the Scientific Literature. Animals (Basel) 3: 882-906. [Crossref]

56. Horwitz DA, Fahmy TM, Piccirillo CA, La Cava A (2019) Rebalancing Immune Homeostasis to Treat Autoimmune Diseases. Trends Immunol 40: 888-908. [Crossref]

57. Kotas ME, Medzhitov R (2015) Homeostasis, inflammation, and disease susceptibility. Cell 160: 816-827. [Crossref]

58. Little JB (1968) Cellular effects of ionizing radiation. $N$ Engl J Med 278: 369-376 concl. [Crossref]

59. Hall EJ, Giaccia AJ (2019) Radiobiology for radiologist. ( $8^{\text {th }}$ edn), Wolters Kluwer.

60. Castro MA, Dalmolin RJS, Moreira JCF, Mombach JCM, de Almeida RMC (2008) Evolutionary origins of human apoptosis and genome-stability gene networks. Nucleic Acids Res 36: 6269-6283.

61. Renehan AG, Booth C, Potten CS (2001) What is apoptosis, and why is it important? $B M J$ 322: 1536-1538. [Crossref]

62. Ghosh S, Indracanti N, Joshi J, Indracanti PK (2017) Rescuing Self: Transient Isolation and Autologous Transplantation of Bone Marrow Mitigates Radiation-Induced Hematopoietic Syndrome and Mortality in Mice. Front Immunol 8: 1180. [Crossref]

63. Ameisen JC (2002) On the origin, evolution, and nature of programmed cell death: a timeline of four billion years. Cell Death Differ 9: 367-393. [Crossref]

64. Allocati N, Masulli M, Di Ilio C, De Laurenzi V (2015) Die for the community: an overview of programmed cell death in bacteria. Cell Death Dis 6: e1609. [Crossref]

65. Bayles KW (2014) Bacterial Programmed Cell Death: Making Sense of a Paradox. Nat Rev Microbiol 12: 63-69.

66. Radman M (1975) Phenomenology of an inducible mutagenic DNA repair pathway in Escherichia coli: SOS repair hypothesis. Basic Life Sci 5: 355-367.

67. Nelson DL, Cox MM (2005) Lehninger: Principles of Biochemistry. (4 $4^{\text {th }}$ edn), W.H. Freeman and Company. New York, USA. pp: 1098.

68. Erental A, Sharon I, Engelberg-Kulka H (2012) Two Programmed Cell Death Systems in Escherichia coli: An Apoptotic-Like Death Is Inhibited by the mazEF-Mediated Death Pathway. PLoS Biol 10: e1001281.

69. Robinson R (2012) In E. coli, Interrupting One Death Pathway Leads You Down Another. PLoS Biol 10: e1001278.

70. Gould SE (2012) The Bacteria that Commit Honourable Suicide. Sci American Available from: https://blogs.scientificamerican.com/lab-rat/the-bacteria-that-commithonourable-suicide/

71. Galluzzi L, Vitale I, Aaronson SA, Abrams JM, Adam D, et al. (2018) Molecular mechanisms of cell death: recommendations of the Nomenclature Committee on Cell Death 2018. Cell Death Differ 25: 486-541.
72. Lei P, Bai T, Sun Y (2019) Mechanisms of ferroptosis and relations with regulated cell death: A review. Front Physiol 10: 139.

73. Williams JP, McBride WH (2011) After the bomb drops: a new look at radiationinduced multiple organ dysfunction syndrome (MODS). Int J Radiat Biol 87: 851-868.

74. Fauchon C, Faillenot I, Quesada C, Meunier D, Chouchou F, et al. (2019) Brain activity sustaining the modulation of pain by empathetic comments. Sci Rep 9: 8398 .

75. Levy J, Goldstein A, Feldman R (2019) The neural development of empathy is sensitive to caregiving and early trauma. Nat Commun 10: 1905.

76. Frank ET, Schmitt T, Hovestadt T, Mitesser O, Stiegler J, et al (2017) Saving the injured: Rescue behavior in the termite-hunting ant Megaponera analis. Sci Adv 3: e1602187.

77. Sparrow, AH, Miksche JP (1961) Correlation of nuclear volume and DNA content with higher plant tolerance to chronic radiation. Science 134: 282-283.

78. Shanks N, Greek R, Greek J (2009) Are animal models predictive for humans? Philos Ethics Humanit Med 4: 2

79. Trowell OA (1952) The sensitivity of lymphocytes to ionising radiation. J Pathol Bacteriol 64: 687-704

80. Moras M, Lefevre SD, Ostuni MA (2017) From Erythroblasts to Mature Red Blood Cells: Organelle Clearance in Mammals. Front Physiol 8: 1076.

81. Brabletz T (2018) EMT in cancer. Nat Rev Cancer 18: 128-134.

82. Singh VK (2014) Radiation countermeasure agents: an update (2011-2014). Expert Opin Ther Patents 24: 12291255.

83. Ariyasu S (2014) Design and synthesis of 8-hydroxyquinoline-based radioprotective agents. Bioorg Med Chem 22: 3891-905.

84. Joshi J (2015) Ligand and Structure Based Models for the Identification of Beta 2 Adrenergic Receptor Antagonists. Curr Comput Aided Drug Des 11: 222-236.

85. Wishart DS (2018) DrugBank 5.0: a major update to the DrugBank database for 2018 Nucleic Acids Res 4: 46.

86. Di Maggio FM (2015) Portrait of inflammatory response to ionizing radiation treatment J Inflamm (Lond) 12: 14.

87. Indraganti PK, Namita I, Anoushka K (2019) Possible role of artificial intelligence in radioprotection. Frontiers Drug Chem Clin Res 2: 1-2.

88. Miller DD, Brown EW (2018) Artificial Intelligence in Medical Practice: The Question to the Answer? Am J Med 131: 129-133.

89. Reichstein M (2019). Deep learning and process understanding for data-driven Earth system science. Nature 566: 195-204.

90. Tortora GJ, Derrickson B (2017) Principles of anatomy \& physiology. John Wiley \& Sons, Inc., Hoboken, New Jersey, USA.

Copyright: (C2019 Indraganti PK. This is an open-access article distributed under the terms of the Creative Commons Attribution License, which permits unrestricted use, distribution, and reproduction in any medium, provided the original author and source are credited. 